\title{
Capacidad antioxidante del fruto de la Opuntia apurimacensis (ayrampo) y de la Opuntia ficus-indica
}

(tuna)

\author{
Antioxidant properties of the fruits Opuntia apurimacensis (ayrampo) \\ and Opuntia ficus-indica (cactus pear)
}

\author{
Paola Jorge ${ }^{1,2}$, Luzmila Troncoso ${ }^{3}$ \\ 'Magister en Ciencias de la Educación, Profesora Auxiliar, Facultad de Ciencias - Universidad Nacional Agraria La Molina, Lima, Perú. \\ ${ }^{2}$ Egresada de la Maestría en Bioquímica, Facultad de Medicina, Universidad Nacional Mayor de San Marcos, Lima, Perú. \\ ${ }^{3}$ Doctora en Medicina, Profesora Principal, Investigadora Permanente, Laboratorio de Bioquímica Clínica y Nutricional, Centro de \\ Investigación de Bioquímica y Nutrición - Facultad de Medicina, Universidad Nacional Mayor de San Marcos, Lima, Perú.
}

\begin{abstract}
Resumen
Introducción. En la tuna existen compuestos antioxidantes, pero, se desconoce si hay en el ayrampo. Objetivo. Determinar la capacidad antioxidante de Opuntia apurimacensis (ayrampo) y de Opuntia ficus-indica (tuna). Diseño. Estudio analítico observacional. Lugar. Laboratorio de Bioquímica Clínica y Nutricional, Centro de Investigación de Bioquímica y Nutrición, Facultad de Medicina, Universidad Nacional Mayor de San Marcos, Lima, Perú. Material biológico. Fruta fresca (ff) de Opuntia apurimacensis (ayrampo) y Opuntia ficusindica (tuna). Intervenciones. En los extractos acuosos se realizaron determinaciones de vitamina C, polifenoles totales y capacidad antioxidante (FRAP). Los resultados se analizaron mediante la prueba de Mann-Whitney, con 95\% de confianza. Principales medidas de resultados. Concentración de vitamina $\mathrm{C}$ y polifenoles totales; capacidad antioxidante. Resultados. El ayrampo presentó mayor concentración de vitamina C (49,9 y 36,1 mg de ácido ascórbico/100 g ff), mayor concentración de polifenoles totales (107,3 y $68,7 \mathrm{mg}$ de equivalente ácido gálico/100 g ff) y mayor capacidad antioxidante (1,1 y 0,7 mmoles de Fe- $1 / / 100 \mathrm{~g} f f)$ que la tuna. E ayrampo mostró mayor correlación entre la capacidad antioxidante y la vitamina $C(0,91$ y 0,56$)$, mientras que, la tuna tuvo mayor correlación entre la capacidad antioxidante y los polifenoles totales $(0,98$ y 0,82). Conclusiones. El ayrampo presentó mayor capacidad antioxidante que la tuna.
\end{abstract}

Palabras clave. Ayrampo; Tuna; Vitamina C; Polifenoles totales; Capacidad Antioxidante.

\section{Abstract}

Introduction. The cactus pear contains antioxidant compounds, but it is unknown if these exist in the ayrampo. Objective. To determine the antioxidant capacity of Opuntia apurimacensis (ayrampo) and Opuntia ficus-indica (cactus pear). Design. Analytical observational study. Location. Laboratory of Clinical Biochemistry and Nutrition, Research Center of Biochemistry and Nutrition, Faculty of Medicine, Universidad Nacional Mayor de San Marcos, Lima, Peru. Biological material. Fresh fruit (ff) of both Opuntia apurimacensis (ayrampo) and Opuntia ficus-indica (cactus pear). Interventions. Determination of vitamin C, polyphenols and antioxidant capacity (FRAP) were performed in both fruits aqueous extracts. Results were analyzed using the Mann-Whitney $U$ test with $95 \%$ confidence. Main outcome measures. Concentration of vitamin C and polyphenols; antioxidant capacity (FRAP). Results. Compared to the cactus pear, ayrampo presented the highest concentration of vitamin C (49.9 and $36.1 \mathrm{mg}$ ascorbic acid/100 g ff), highest concentration of total polyphenols (107.3 and $68.7 \mathrm{mg}$ gallic acid equivalent/100 g ff) and highest antioxidant capacity (1.1 and $0.7 \mathrm{mmol}$ of Fe-II/100 g ff). Ayrampo showed the highest correlation between antioxidant capacity and vitamin $\mathrm{C}(0.91$ and 0.56$)$, and the prickly pear the higher correlation between antioxidant capacity and total polyphenols (0.98 and 0.82 ). Conclusions. Ayrampo showed higher antioxidant capacity compared to cactus pear.

Keywords. Ayrampo; Cactus Pear; Vitamin C; Total Polyphenols; Antioxidant Capacity.

An Fac med. 2016;77(2):105-9 / http://dx.doi.org/10.15381/anales.v77i2.11812 


\section{INTRODUCCIÓN}

Estudios epidemiológicos demuestran que el consumo regular de frutas y verduras está asociado con la disminución del riesgo de padecer enfermedades crónicas como cáncer, problemas cardiovasculares, Alzheimer, derrames cerebrales, cataratas o el deterioro funcional asociado a la edad ${ }^{(1)}$. Por tanto, un aumento en la ingesta de estos antioxidantes dietarios podrían proteger de estas enfermedades crónicas.

La acción antioxidante que tienen estas frutas es importante ante el estrés oxidativo que se presenta en el organismo humano, cuando los sistemas antioxidantes (enzimático y no enzimático) no son suficientes para proteger de la acción dañina de los radicales libres ${ }^{(2)}$. Estos radicales libres son generados por el mismo organismo, incrementando su acción nociva mediante factores externos como son los contaminantes ambientales, farmacológicos y nutricionales ${ }^{(3)}$.

Los radicales libres tienen la propiedad de reaccionar con proteínas, carbohidratos, lípidos y ácidos nucleicos, reacción asociada a múltiples enfermedades crónicas no transmisibles (2). El consumo de frutas con capacidad antioxidante es utilizado con el objetivo de prevenir dichas enfermedades crónicas ${ }^{(1,2,4,5)}$.

El género Opuntia ha sido ampliamente utilizado en la medicina popular, en alimentos y en el campo farmacéutico y ha demostrado ser rico en compuestos antioxidantes, tales como polifenoles, betalaínas ${ }^{(5)}$ y vitamina $\mathrm{C}^{(1)}$. Además, es una fuente natural de minerales ( $\mathrm{K}, \mathrm{Ca}, \mathrm{Na}, \mathrm{Fe}, \mathrm{Mg}, \mathrm{Mn}$, Zn), ácidos orgánicos (málico, oxálico y otros), aminoácidos (alanina, arginina, asparagina, ácido glutámico, glutamina, histidina, metionina, prolina, serina, valina y otros en menores cantidades), y rico en azúcares ${ }^{(6)}$. Estos componentes químicos le dan un valor agregado a los frutos de opuntia en base a su funcionalidad nutricional.
La especie Opuntia ficus-indica, de nombre vulgar tuna, probablemente proviene de México y de las Islas del Caribe y la Opuntia apurimacensis, de nombre vulgar ayrampo, se cree que tiene el mismo origen que la Opuntia ficus-indica. La tuna presenta tres variedades: roja, verde y amarilla; pero, el ayrampo, solo la de color morado. La tuna se cultiva en varias regiones del Perú, incluida Lima, y el ayrampo se distribuye en la Región de Apurímac, entre Andahuaylas y Abancay ${ }^{(7)}$. A partir de la tuna se elaboran jugos, mieles, queso, melcocha, colonche, jaleas y vino de buena calidad; asimismo, se puede elaborar colorantes; además, es usada como planta ornamental y hospedera de la cochinilla ${ }^{(7)}$. No se tiene información documentada sobre los usos del ayrampo, pero se conoce que es utilizado para preparar chicha, mazamorra, acompañante del cañazo, como medicina natural para bajar la fiebre y evitar el rebrote de las enfermedades eruptivas, en el Distrito de San Miguel de Mayocc.

El objetivo del presente trabajo fue determinar la capacidad antioxidante de la Opuntia apurimacensis (ayrampo) y de la Opuntia ficus-indica (tuna), provenientes del departamento de Huancavelica.

\section{MÉTODOS}

Los frutos frescos (ff) de Opuntia ficusindica (L.) Miller, de nombre vulgar tuna, variedad roja, y de Opuntia apurimacensis (F. Ritter) R. Crook \&ु Mottram, de nombre vulgar ayrampo, procedieron del Distrito de San Miguel de Mayocc del Departamento de Huancavelica. La clasificación botánica fue realizada por la Dra. Mónica Arakaki, en el Herbario del Museo de Historia Natural de la Universidad Nacional Mayor de San Marcos.

El reactivo 2,4,6-tris (2-piridil)-striazina se adquirió de Sigma Chemical Company y los reactivos Folin-Ciocalteau, ácido tricloroacético, carbonato de sodio, tricloruro de hierro, ácido acético, ácido gálico, acetato de sodio, ácido clorhídrico y cloruro férrico se adquirieron de Merck Darmstadt. Todos ellos fueron de grado para análisis.

Para la preparación del extracto acuoso se usó la pulpa de los frutos frescos de tuna y ayrampo, se procedió a licuar y filtrar con papel whatman №2, obteniendo una concentración de 25 g en $100 \mathrm{~mL}$ de agua destilada para la tuna y $3 \mathrm{~g}$ en $100 \mathrm{~mL}$ de agua destilada para el ayrampo. El extracto acuoso se preparó en cinco oportunidades y cada tubo se trabajó por triplicado. El peso promedio de la tuna fue $155,9 \pm 4,4 \mathrm{~g}$ y del ayrampo $15,9 \pm 0,5 \mathrm{~g}$.

Con respecto a la determinación de vitamina $C$, se utilizó la técnica propuesta por Jagota y Dani ${ }^{(8)}$. Para un volumen final de $2,0 \mathrm{~mL}$ se utilizó, independientemente, $0,1 \mathrm{~mL}$ del extracto acuoso de la tuna y el doble de volumen para el ayrampo; ácido tricloroacético al 10\%; reactivo de Folin-Ciocalteu 2 M diluido 1:10. Se dejó en reposo durante 10 minutos a temperatura ambiente, a cuyo término se leyó a $760 \mathrm{~nm}$ en el espectrofotómetro. Se preparó el blanco correspondiente y una curva estándar utilizando concentraciones de 0,2 a 2,5 mg/dL de ácido ascórbico.

En relación a la determinación de polifenoles totales, se utilizó el método de Singleton, Orthofer y LamuelaRaventos ${ }^{(9)}$. Se empleó 0,1 mL del extracto acuoso de la tuna y el doble de volumen para el ayrampo, reactivo de Folin-Ciocalteu diluido 1:10 y una solución de carbonato de sodio al 7,5\%, en cantidad suficiente para un volumen final de 2,0 mL. Los tubos fueron colocados en baño maría a $45^{\circ} \mathrm{C}$ durante 15 minutos, a cuyo término se leyeron a $765 \mathrm{~nm}$ en el espectrofotómetro. Paralelamente, se preparó el blanco correspondiente y para realizar los cálculos se usó una curva estándar utilizando concentraciones de 0,1 a $2,5 \mathrm{mg} / \mathrm{dL}$ equivalentes de ácido gálico.

Para la determinación de la capacidad antioxidante, se usó la técnica descrita por Benzie y Strain, adaptada por Szollosi y Varga (FRAP) ${ }^{(10)}$. El reacti- 
Tabla 1. Concentraciones de vitamina C, polifenoles totales y capacidad antioxidante de los frutos de tuna y ayrampo.

\begin{tabular}{lccc} 
Variables & Tuna & Ayrampo & Valor $p$ \\
Vitamina C (mg de ácido ascórbico en $100 \mathrm{~g}$ de ff) & $36,1 \pm 1,7$ & $49,9 \pm 6,3$ & 0,000 \\
Polifenoles totales (mg equivalentes de ácido gálico en 100 g de ff) & $68,7 \pm 2,3$ & $107,3 \pm 10,6$ & 0,000 \\
FRAP (mmoles de Fe-II en $100 \mathrm{~g}$ de ff) & $0,7 \pm 0,0$ & $1,1 \pm 0,1$ & 0,000 \\
Contribución de vitamina C a la capacidad antioxidante (porcentaje) & 76,8 & 68,5 & \\
\hline
\end{tabular}

Datos expresados en promedios con desviación estándar.

vo FRAP se preparó mezclando buffer acetato de sodio ( $\mathrm{pH} 3,6), 2,4,6$-tris (2piridil)-s-triazina y cloruro férrico. $\mathrm{La}$ determinación se realizó con el reactivo FRAP, $25 \mu \mathrm{L}$ del extracto acuoso de la tuna y el doble de volumen para el ayrampo, en un volumen final de 2,0 $\mathrm{mL}$. Se preparó el blanco respectivo y todos los tubos fueron colocados en baño maría a $37^{\circ} \mathrm{C}$ durante 15 minutos, a cuyo término se leyó a $593 \mathrm{~nm}$ en el espectrofotómetro. Se preparó una curva estándar utilizando concentraciones de 5 a $50 \mu$ moles $/ \mathrm{L}$ de sulfato ferroso que sirvió para realizar los cálculos.

En relación al cálculo de la contribución de la vitamina $\mathrm{C}$ a la capacidad antioxidante, se preparó una curva estándar entre el ácido ascórbico y su correspondiente capacidad antioxidante. Las concentraciones de ácido ascórbico estuvieron comprendidas entre 60 y $140 \mathrm{mg} / \mathrm{dL}$. Se halló la cantidad de mmoles de sulfato ferroso a partir de la concentración de ácido ascórbico presente en la fruta, haciendo uso de la curva. Luego se calculó el porcentaje de capacidad antioxidante entre el valor que presentó la fruta y el valor hallado en la curva.

Todos los resultados se analizaron mediante estadísticos descriptivos como promedio y desviación estándar e inferencial como la prueba de MannWhitney.

Se evaluó la distribución normal de todos los resultados a través de la prueba de Shapiro-Wilk y Anderson-Darling. Se utilizó la prueba no paramétrica de Mann-Whitney, cuando por lo menos uno de los grupos no cumplía con la normalidad, teniendo como objetivo comprobar las diferencias estadística- mente significativas entre las medias de los grupos, con un alfa igual a 0,05. Además se determinó el coeficiente de correlación de Pearson para mostrar la relación entre la capacidad antioxidante de las muestras biológicas y sus componentes bioactivos como vitamina $\mathrm{C} y$ polifenoles totales. Así mismo, se aplicó el diagrama de cajas y bigotes para todos los resultados de cada grupo.

Los resultados fueron procesados por el programa Minitab v17 y XLSTAT.

\section{RESULTADOS}

En el extracto acuoso de tuna y ayrampo se determinó la concentración de componentes bioactivos como la vitamina $\mathrm{C}$ y polifenoles totales, siendo en ambos casos, los valores de ayrampo aproximadamente 1,5 veces superiores al de la tuna. Así mismo, se estableció la capacidad antioxidante en estos dos extractos acuosos, siendo los valores del ayrampo 1,5 veces superiores al de la tuna. Además, se halló la contribución de la vitamina $\mathrm{C}$ a la capacidad antioxidante (tabla 1).

Los resultados fueron analizados mediante la prueba de Mann-Whitney y se obtuvo diferencia estadísticamente significativa en las tres determinaciones, con un p-valor de 0,000 .

Tabla 2. Coeficientes de correlación de Pearson entre los componentes bioactivos (vitamina C y polifenoles totales) y la capacidad antioxidante (FRAP) en frutos de tuna y ayrampo.

\begin{tabular}{ccc} 
Fruta & \multicolumn{2}{c}{ Correlaciones } \\
\cline { 2 - 3 } & Vitamina C - FRAP & Polifenoles totales - FRAP \\
Tuna & 0,56 & 0,98 \\
Ayrampo & 0,91 & 0,82 \\
\hline
\end{tabular}

Mediante los resultados obtenidos, se halló el coeficiente de correlación de Pearson entre los componentes bioactivos (vitamina $\mathrm{C}$ y polifenoles totales) y la capacidad antioxidante (tabla 2).

\section{DISCUSIÓN}

La concentración de vitamina C (tabla 1) en la tuna, variedad roja, estuvo dentro del promedio de los valores esperados de 22,8 a $45,8 \mathrm{mg}$ de ácido ascórbico en $100 \mathrm{~g}$ de fruta fresca ${ }^{(4,5,11)}$. La concentración hallada para el ayrampo fue superior a muchas frutas, como el aguaymanto, la papaya de monte, el tomate de árbol, con 43,3, 31,4 y 16,1 mg de ácido ascórbico en $100 \mathrm{~g}$ de fruta fresca, respectivamente ${ }^{(11)}$; la palta y el sapote negro con 39,9 y $24,1 \mathrm{mg}$ de ácido ascórbico en $100 \mathrm{~g}$ de fruta fresca, respectivamente ${ }^{(4)}$, incluyendo a la tuna presentado por diferentes autores ${ }^{(4,5,11)}$.

En relación a la concentración de polifenoles totales (tabla 1), la tuna, variedad roja, estuvo dentro de los valores esperados de 52,0 a $72,3 \mathrm{mg}$ de equivalente de ácido gálico en $100 \mathrm{~g}$ de fruta fresca ${ }^{(4,11,12)}$. La concentración para el ayrampo fue superior a frutas como la papaya, de 53,8 mg de equivalente ácido gálico en $100 \mathrm{~g}$ de fruta fresca ${ }^{(4)}$, la piña, la guayaba y guaná- 


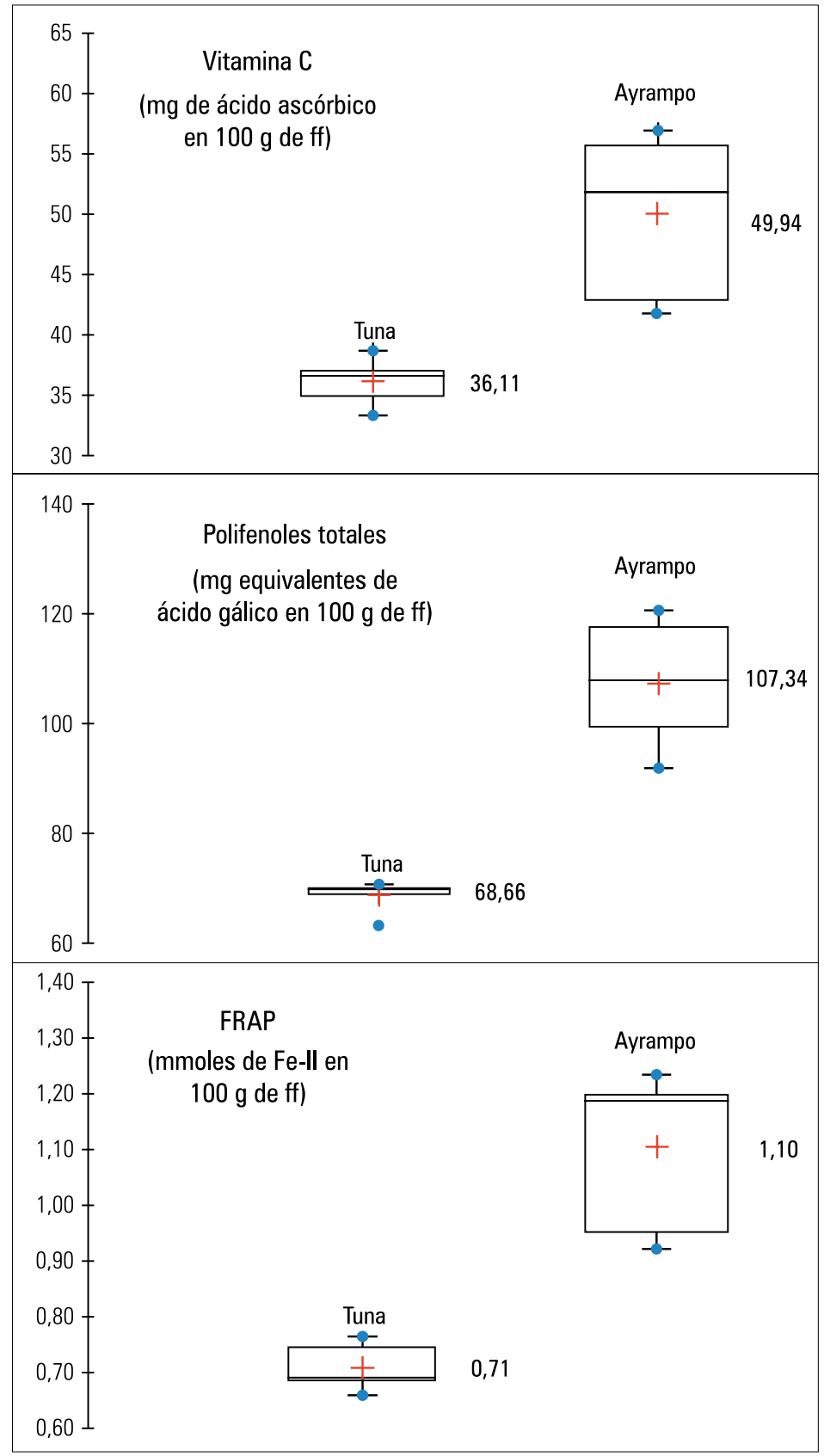

Figura 1. Diagrama de cajas de las concentraciones de vitamina C, polifenoles totales y capacidad antioxidante de los frutos de tuna y ayrampo. bana que presentan $8,9,56,9,39,6 \mathrm{mg}$ equivalentes de ácido gálico en $100 \mathrm{~g}$ de fruta fresca, respectivamente ${ }^{(12)}$, incluyendo a la tuna como reportado por diferentes autores ${ }^{(4,11,12)}$.

Según lo hallado, el ayrampo mostró una alta concentración de polifenoles totales, los que están directamente relacionados con las propiedades organolépticas del alimento, como sabor, aroma, astringencia y color ${ }^{(13)}$. Los compuestos polifenólicos constituyen un grupo importante de antioxidantes naturales capaces de neutralizar radicales libres, recomendándose su consumo para la prevención de enfermedades crónicas ${ }^{(1)}$.

El valor de la capacidad antioxidante hallado para la tuna, variedad roja (tabla 1), estuvo dentro de los valores reportados para diferentes frutas, de 0,4 a 56,0 mmoles de Fe-II en $100 \mathrm{~g}$ de fruta fresca correspondiente a la toronja y a la uva roja rose, respectivamente ${ }^{(15)}$, y también dentro de los valores reportados para diferentes verduras, de 0,1 a 2,7 mmoles de Fe-II en $100 \mathrm{~g}$ de peso fresco correspondiente al pepino y a la espinaca, respectivamente ${ }^{(16)}$.

Se obtuvo un valor alto de capacidad antioxidante para el ayrampo en relación al de la tuna (figura 1) y este valor de la capacidad antioxidante guardó relación directa con el contenido de sus componentes bioactivos (vitamina $\mathrm{C}$ y polifenoles totales). Este comportamiento se explica porque la capacidad antioxidante de un alimento se debe a la actividad antioxidante de sus diferentes compuestos, tales como los polifenoles, carotenos, antocianinas, ácido ácido ascórbico y otros, los que tendrían un efecto sinérgico ante la capacidad antioxidante del fruto ${ }^{(11)}$. En ambos casos se comprobó que la capacidad antioxidante de los frutos de opuntia provinieron de la vitamina C, al ser mayor a un $50 \%$, siendo la contribución de la vitamina $\mathrm{C}$ a la capacidad antioxidante de la tuna ligeramente mayor que la del ayrampo (ver tabla 1).

En la tabla 2 se observa los valores de los coeficientes de correlación entre 
componentes bioactivos (vitamina $\mathrm{C} y$ polifenoles totales) y la capacidad antioxidante en frutos de tuna y ayrampo. Según los resultados hallados, hubo una mayor relación entre los polifenoles totales y la capacidad antioxidante que entre la vitamina $\mathrm{C}$ y la capacidad antioxidante en la tuna. Resultados similares se halló en el estudio sobre la correlación entre componentes nutricionales y capacidad antioxidante total en cultivos hortícolas ${ }^{(4)}$. En otra investigación sobre propiedades antioxidantes de clones de opuntia spp. Se mencionó que los principales principios activos en tunas fueron los compuestos fenólicos, seguido por pigmentos como la betacianina y betaxantina y, por último, el ácido ascórbico ${ }^{(14)}$.

Además, se menciona que la tuna tiene mayor capacidad antioxidante proveniente de componentes hidrosolubles que los liposolubles, es decir, que los polifenoles totales y vitamina $\mathrm{C}$ presentan mayor capacidad antioxidante que los carotenoides totales, beta caroteno y vitamina $\mathrm{E}^{(4)}$.

La tuna, variedad roja, tuvo una alta actividad antioxidante como consecuencia de su elevada concentración de polifenoles, betalaínas y vitamina $\mathrm{C}^{(4,5,13,17)}$; por ende, el ayrampo también mostró una alta actividad antioxidante, siendo superior al de la tuna, ya que los valores de sus componentes bioactivos fueron mayores.

Las diferencias de las concentraciones de vitamina $\mathrm{C}$ y polifenoles totales entre las especies y cultivares podrían deberse a las condiciones de cultivo, ya que el género Opuntia al crecer en condiciones limitadas de suelo y agua, podría modificar su composición, principalmente la del fruto ${ }^{(18)}$.

En conclusión, el ayrampo presentó mayor capacidad antioxidante que la tuna, debido al mayor contenido de sus componentes bioactivos, tales como vitamina $\mathrm{C}$ y polifenoles totales.

\section{AGRADECIMIENTOS}

Al Doctor Emilio Guija Poma por sus valiosos consejos y a la Mg. Gisela Oliveira Bardales por su apoyo incondicional.

\section{REFERENCIAS BIBLIOGRÁFICAS}

1. Tesoriere L, Butera D, Pintaudi A, Allegra M, Livrea M. Supplementation with cactus pear (Opuntia ficus-indica) fruit decreases oxidative stress in healthy humans: a comparative study with vitamin C. Am J Clin Nutr. 2004 Aug;80:391-5.

2. Reátegui O, Guija E, Soldevilla P, Castillo M Pérez S. Determinación de la capacidad antioxidante de néctares de frutas. Revista Científica. 2008;5(6):19-26.

3. López A. Efecto del zumo de mandarina sobre el estrés oxidativo: estudio experimental y en patologias mediadas por radicales libres [Tesis Doctoral]. Valencia - España: Universidad de Valencia; 2014: 287 pp.

4. Corral R, Yahia E, Carrillo A, Gonzáles G. Correlation between some nutritional components and the total antioxidant capacity measured with six different assays in eight horticultural crops. J Agric Food Chem. 2009 Nov 26;56(22):10498-504. doi: $10.1021 / \mathrm{jf} 801983 \mathrm{r}$.

5. Kuti J. Antioxidant compounds from four Opuntia cactus pear fruit varieties. Food Chem 2004 May;85:527-33. doi:10.1016/S03088146(03)00184-5.

6. Rodrigo G. Actividad genotóxica de Opuntia soehrensii, evaluada por el test de mutación y recombinación somática en $D$. melanogaster. Biofarbo. 2007;15(1):61-6.

7. Ostolaza C. Todos los Cactus del Perú. Primera edición. Lima - Perú: Editorial Franco EIRL. 2004:194-6.

8. Jagota SK, Dani HM. A new colorimetric technique for the estimation of vitamin C using Folin pheno reagent. Anal Biochem. 1982;127(1):178-82. doi: 10.1016/0003-2697(82)90162-2.

9. Singleton V, Orthofer R, Lamuela-Raventos R Analysis of total phenols and other oxidation substrates and antioxidants by means of Folin-Ciocalteau reagent. Methods Enzymol. 1999;299(1):15278. doi: 10.1016/S0076-6879(99)99017-1.

10. Szollosi R, Varga I. Total antioxidant power in some species of Labiatae (Adaptation of FRAP method). Acta Biol Szeged. 2002;46(3-4):125-7.

11. Repo R, Encina C. Determinación de la capacidad antioxidante y compuestos bioactivos de frutas nativas peruanas. Rev Soc Quim Perú. 2008;74(2):108-24
12. Alba J, Chávez J, Verdalet I, Martínez A, Aquino E. Betalainas, polifenoles y actividad antioxidante en tuna roja minimamente procesada, almacenada en atmósferas controladas. Gayana Bot. 2014;71(2):222-6.

13. Yahia E, Mondragón C. Nutritional components and anti-oxidant capacity of ten cultivars and lines of cactus pear fruit (Opuntia spp.). Food Res Int. 2011 Feb;44:2311-8. doi: 10.1016/j. foodres.2011.02.042.

14. Stintzing FC, Herbach KM, Mosshammer MR, Carle R, Yi W, Sellappan S, Akoh CC, Bunch R, Felker P. Color, betalain pattern, and antioxidant properties of cactus pear (Opuntia spp.) clones. J Agric Food Chem. 2005 Jan 26;53(2):442-51.

15. Guo Ch, Yanga J, Weia J, Lia Y, Xua J, Jianga $Y$. Antioxidant activities of peel, pulp and seed fractions of common fruits as determined by FRAP assay. Nutr Res. 2003 Dec;23(12):1719-26. doi:10.1016/j.nutres.2003.08.005.

16. Sun $T$, Tanumihardjo $S$. An integrated approach to evaluate food antioxidant capacity. J Food Sci. 2007 Dec;72(9):159-65. doi:10.1111/j.17503841.2007.00552.x.

17. Tesoriere L, Fazzari M, Allegra M, Livrea M. Biothiols, taurine, and lipid-soluble antioxidants in the edible pulp of Sicilian cactus pear (Opuntia ficus-indica) fruits and changes of bioactive juice components upon industrial processing. J Agric Food Chem. 2005 Oct 5;53(20):7851-5.

18. Figueroa I, Martínez M, Rodriguez E, Colinas M, Valles S, Ramírez S, Gallegos C. Contenido de pigmentos, otros compuestos y capacidad antioxidante en 12 cultivares de tuna (Opuntia spp.) de México. Agrociencia. 2010;44:763-71.

Apoyo recibido del Laboratorio de Bioquímica Clínica y Nutricional, Centro de Investigación de Bioquímica y Nutrición, Facultad de Medicina, Universidad Nacional Mayor de San Marcos.

\section{Conflicto de interés:}

No existe conflicto de interés.

Trabajo ganador de la sección Estudiantes de la XIV Jornadas Cientificas Sanfernandinas 2015, XVII Jornadas de Investigación en Salud, XXIV Jornadas Sanfernandinas estudiantiles "Investigación y Medicina Centrada en la Persona", realizadas entre el 7 y 9 de septiembre de 2015.

\section{Correspondencia:}

Paola Jorge Montalvo.

Urb. Mariscal Cáceres Mz. E4 Lote 12 S.J.L.

Teléfono: 990293366

Correo electrónico: paolajom@lamolina.edu.pe 